\title{
Contingent claim valuation with penalty costs on short selling positions
}

\author{
O. L. V. Costa \& E. V. Queiroz Filho \\ Departamento de Engenharia de Telecomunicações e Controle, \\ Escola Politécnica da Universidade de São Paulo, Brazil
}

\begin{abstract}
In this paper we consider a discrete-time finite sample space financial model with penalty costs on short selling positions. We start by presenting a necessary and sufficient condition for the non-existence of arbitrage opportunities. This reduces to the existence of a martingale measure for the case in which the penalties are zero. Next we consider the problem of contingent claim valuation. Our main result states that, under certain conditions, for every contingent there will be a seller price and a buyer price, with a perfect portfolio replication for each of them. Again when the penalty costs on short selling positions are zero, our conditions coincide with the traditional condition for the market to be complete. An explicit and constructive procedure for obtaining hedging strategies, not necessarily in the binomial framework, is presented.
\end{abstract}

Keywords: transaction costs, perfect replication, bid and ask option pricing.

\section{Introduction}

The general theory for contingent claim valuation considers that the prices for a buying position and a short selling position in a security are the same. However in practice these values are not the same, due to penalty costs on short selling positions. This penalty can been seen as a premium risk charged on a short selling position or on the way in which the bid and ask process affects the prices.

The subject of pricing derivatives with transaction costs and portfolio selection under transaction costs is of practical importance, and has been in evidence over the last years. Two types of transaction costs are considered; fixed costs, which are paid whenever there is a change of position, and proportional costs, which are charged according to the volume traded. Several different approaches to the prob- 
lem of pricing derivatives with transaction costs and the portfolio choice problem under transaction costs can be found in the literature. In Edirisinghe et al. [1], the authors approach the problem by finding the least-cost replication strategy for hedging the pay off of contingent claims. In Davis et al. [2] the authors state the problem as an stochastic optimal control problem. In Leland [3] the author presents an alternative replicating strategy which depends on the size of transaction costs and frequency of revisions. As a result it presents a modified volatility that is incorporated in the Black-Scholes formula. In Boyle and Vorst [4] the authors construct a replicating strategy for the call option by embedding a given binomial model with proportional transaction costs into a complete Cox-RossRubinstein [5] model with such costs. This approach can be seen as a discretetime variant of the result derived by Leland [3], and was somewhat extended in Melnikov and Petrachenko [6]. Several other papers studied the problem considering, for instance, the theory of cones, mean-variance techniques, minimizing an expected discount loss function, etc (see, for instance, [7-10]). In comparison to these papers, our work gives an explicit and constructive procedure for obtaining hedging strategies, not necessarily in the binomial framework.

In this paper we consider a discrete-time finite sample space financial model with different prices for a buying and short selling position in the value of the portfolio. This is done by introducing penalty factors for the short selling position. Due to this, strategies are broken into $\mathrm{H}_{i}^{+}$and $H_{i}^{-}$, denoting the long and short positions respectively. In additional we introduce the concept of maximal trading strategy (see Definition 2.2). Moreover, unlike the standard theory, in our case the non-existence of arbitrage does not necessarily implies the non-existence of dominant strategies neither that the law of one price holds (see Pliska [11], p.10). Our definition of contingent claim consistently realizable (see Definition 2.7) rules out these situations, so that logical pricing can be obtained.

The paper is organized in the following way. Section 2 presents the model, the main definitions, and some preliminary results. In section 3 we present some results and definitions for the single -period case. In section 4 we present the multiperiod case with an special attention to Theorem 4.3 that defines an algorithm to pricing contingent claims under penalty costs. The paper is concluded in section 5 analyzing the binomial case.

\section{Definitions and preliminaries}

Let $m$ be a positive integer. The real $m$-dimensional vector space will be denoted by $\mathbb{R}^{m}$ and for $x \in \mathbb{R}^{m}$ we shall write $x_{i}$ for the $i^{t h}$ component of the vector $x$. We write $x \geq 0$ to denote that all components of $x$ are positive, that is, $x_{i} \geq 0$ for $i=1, \ldots, m$. The transpost of a vector or a matrix will be denoted by $/$. The vector formed by 1 in all components will be represented by $e$, and the vector with 1 in the $i^{t h}$ component and 0 elsewhere by $b^{i}$. For a finite sample space $\Omega$ define $\mathbb{P}(\Omega)$ as the set of probability measures over $\Omega$.

Let $\kappa$ and $N$ be positive integer numbers. We consider the following elements for the financial market. 
i) Initial date $t=0$ and terminal date $t=T$, with trading possible at any time $t$ between these two dates.

ii) A finite sample space $\Omega$ with $\kappa$ elements, that is, $\Omega=\left\{\omega_{1}, \ldots, \omega_{\kappa}\right\}$.

iii) A probability $P \in \mathbb{P}(\Omega)$ with $P(\omega)>0$ for each $\omega \in \Omega$.

iv) A bank account process $B(t), t=0, \ldots, T$, with $B(0)=1$, and $B(t)$, $t=1, \ldots, T$, random variables on $\Omega$ with $B(t) \geq 1$.

v) A price process $S=\{S(t) ; t=0, \ldots, T\}$, where $S(t)$ are $N$-dimensional positive random variables on $\Omega$. $S_{i}(t)$ represents the price value of the $i^{t h}$ security at time $t$.

vi) A filtration $\mathcal{F}=\left\{\mathcal{F}_{t} ; t=0, \ldots, T\right\}$ where each $\mathcal{F}_{t}$ represents the $\sigma$-field generated by the random vectors $\{S(0), \ldots, S(t)\}$ and random variables $\{B(0), \ldots, B(t)\}$.

vii) The penalty cost factors $\left\{\alpha_{i}(t) ; i=0,1, \ldots, N, t=0,1, \ldots, T\right\}$. These factors are related to the penalty costs that should be paid when holding a short selling position. When they are zero, there is no penalty cost, and the model reduces to the standard model.

Now, define the discounted price process $S^{*}=\left\{S^{*}(t) ; t=0, \ldots, T\right\}$ as: $S_{i}^{*}(t):=\frac{S_{i}(t)}{B(t)}, i=1 \ldots, N$; and for $t=0, \ldots, T$, define $\Delta B(t):=B(t)-$ $B(t-1), \Delta S(t):=S(t)-S(t-1)$ and $\Delta S^{*}(t):=S^{*}(t)-S^{*}(t-1)$.

A trading strategy $H=(H(1), \ldots, H(T))$ describes an investor's portfolio from time $t=0$ up to time $t=T$. Each $H(t)$ is a $(N+1,2)$-dimensional random matrix with all components positive. Here it will be more convenient to represent the components of $H(t)$ as follows: $H_{i}^{+}(t) \geq 0, i=0, \ldots, N$ denotes the $N+1$ components of the first column of the matrix $H(t)$, and $H_{i}^{-}(t) \geq 0, i=0, \ldots, N$ denotes the $N+1$ components of the second column of the matrix $H(t)$. The elements $H_{i}^{+}(t)$ represent the buying position at the security $i$, while $H_{i}^{i}(t)$ represents the short selling position at the security $i$. We assume that each trading position $H(t), t=1, \ldots, T$, is $\mathcal{F}_{t-1}$-measurable, so that it is established by taking into account only the information available up to time $t-1$.

Associated to a trading strategy $H$ we have the value process $V:=(V(0), \ldots$, $V(T)$ ) describing the total value of the portfolio at each time $t$. This can be written, at time $t=0$, as

$$
V(0)=\left(H_{0}^{+}(1)-H_{0}^{-}(1)\right) B(0)+\sum_{i=1}^{N}\left(H_{i}^{+}(1)-H_{i}^{-}(1)\right) S_{i}(0) .
$$

and at times $t=1, \ldots, T$, as

$$
\begin{aligned}
& V(t)=\left(H_{0}^{+}(t)-H_{0}^{-}(t)\right) B(t)+\sum_{i=1}^{N}\left(H_{i}^{+}(t)-H_{i}^{-}(t)\right) S_{i}(t), \\
& -\left(\alpha_{0} H_{0}^{-}(t) B(t-1)+\sum_{i=1}^{N} \alpha_{i} H_{i}^{-}(t) S_{i}(t-1)\right) .
\end{aligned}
$$


The quantity $V(t)$ represents the value of the portfolio at time $t$ just before any change of ownership positions take place at that time. The penalty costs due to short selling positions are represented by $\alpha_{0} H_{0}^{-}(t) B(t-1)$ and $\alpha_{i} H_{i}^{-}(t) S_{i}(t-1)$. The assumption here is that these costs are fixed at time $t-1$ as a percentual $\left(\alpha_{i}\right)$ of the value of the security $\left(B(t-1)\right.$ or $\left.S_{i}(t-1)\right)$. If no short selling position is hold at the security $i, H_{i}^{-}(t)=0$ and no cost is paid.

The value of the portfolio at time $t+1$ just after the change of ownership positions is

$$
\left(H_{0}^{+}(t+1)-H_{0}^{-}(t+1)\right) B(t)+\sum_{i=1}^{N}\left(H_{i}^{+}(t+1)-H_{i}^{-}(t+1)\right) S_{i}(t) .
$$

We consider in this paper self-financing trading strategies, so that no money is added or withdrawn from the portfolio between times $t=0$ to time $t=T$. Any change in the portfolio's value is due to a gain or loss in the investments, and penalty costs due to the short selling positions. Thus (3) must coincide with $V(t)$, that is,

$$
\begin{aligned}
V(t) & =\left(H_{0}^{+}(t+1)-H_{0}^{-}(t+1)\right) B(t), \\
& +\sum_{i=1}^{N}\left(H_{i}^{+}(t+1)-H_{i}^{-}(t+1)\right) S_{i}(t) .
\end{aligned}
$$

From eqns (1), (2) and (4) we have for $t=0, \ldots, T-1$

$$
\begin{aligned}
& V(t+1)=V(t)+\left(H_{0}^{+}(t+1)-H_{0}^{-}(t+1)\right) \Delta B(t+1), \\
& +\sum_{i=1}^{N}\left(H_{i}^{+}(t+1)-H_{i}^{-}(t+1)\right) \Delta S_{i}(t+1) \\
& -\left(\alpha_{0} H_{0}^{-}(t+1) B(t)+\sum_{i=1}^{N} \alpha_{i} H_{i}^{-}(t+1) S_{i}(t)\right) .
\end{aligned}
$$

We recall that the discounted process $V^{*}(t)$ is defined as: $V^{*}(t):=\frac{V(t)}{B(t)}$. From eqns (1), (2) and (4) we have for $t=0, \ldots, T-1$,

$$
\begin{aligned}
& V^{*}(t+1)=V^{*}(t)+\sum_{i=1}^{N}\left(H_{i}^{+}(t+1)-H_{i}^{-}(t+1)\right) \Delta S_{i}^{*}(t+1), \\
& -\left(\alpha_{0} H_{0}^{-}(t+1) \frac{B(t)}{B(t+1)}+\sum_{i=1}^{N} \alpha_{i} H_{i}^{-}(t+1) \frac{S_{i}(t)}{B(t+1)}\right) .
\end{aligned}
$$

The following proposition is easily shown.

Proposition 2.1 The following assertions are equivalent:

i) the trading strategy $H$ is self-financing; 
ii) the value process $V:=(V(0), \ldots, V(T))$ associated to a trading strategy $H$ satisfies eqns (1) and (5);

iii) the value process $V:=(V(0), \ldots, V(T))$ associated to a trading strategy $H$ satisfies eqns (1) and (6).

From eqn (2) we notice that any reasonable trading strategy $H$ will be such that $H_{i}^{+}(t) \times H_{i}^{-}(t)=0$ since otherwise, the investor would be holding, at the same time, a buying and short selling position at the security $i$, incurring on unnecessary payment of taxes. Therefore we introduce the following definition:

Definition 2.2 We say that the trading strategy $H$ is maximal if $H_{i}^{+}(t) \times H_{i}^{-}(t)=$ 0 for every $i=0, \ldots, N$ and $t=1, \ldots, T$.

We have the following result:

Proposition 2.3 For any self-financing trading strategy $H$ with associated value process $V$ we can define a self-financing maximal trading strategy $\widetilde{H}$ with associated value process $\widetilde{V}$ such that $V(0)=\widetilde{V}(0)$ and $V(t) \leq \widetilde{V}(t)$ for $t=1, \ldots, T$.

Defining an appropriate recursive trading strategy $\widetilde{H}$ it is easy to verify that $H_{i}^{+}(t), H_{i}^{-}(t)$ are $\mathcal{F}_{t-1}$-measurable, $\widetilde{H}_{i}^{+}(t) \times \widetilde{H}_{i}^{-}(t)=0$ so that $\widetilde{H}$ is a maximal trading strategy, $\widetilde{H}$ is self-financing, and that $V(t) \leq \widetilde{V}(t)$, with $V(0)=\widetilde{V}(0)$.

Next we recall the definition of an arbitrage opportunity.

Definition 2.4 We say that there is an arbitrage opportunity if for some selffinancing maximal trading strategy $H$ we have

i) $V(0)=0$,

ii) $V(T) \geq 0$, and

iii) $E(V(T))>0$.

The next proposition shows that we do not need to require the trading strategy to be maximal in the definition of an arbitrage.

Proposition 2.5 There is a arbitrage opportunity if and only if for some selffinancing trading strategy $H$ we have i), ii) and iii) in Definition 2.4 verified.

The main concern of this paper will be the problem of valuation of a contingent claim. We recall (see [11]) that a contingent claim is a random variable $X$ representing a payoff at the final time $T$. We shall need the following definitions.

Definition 2.6 We say that a contingent claim $X$ is realizable if there exists a selffinancing trading strategy $H$ with associated value process $V$ such that $X\left(\omega_{j}\right)=$ $V\left(T, \omega_{j}\right)$ for every $j=1, \ldots, \kappa$. We say in this case that $H$ is a replicating trading strategy for $X$.

Definition 2.7 We say that a contingent claim $X$ is consistently realizable if $X$ is realizable and for any replicating self-financing trading strategy $H$ for $X$ with associated value process $V$ and any self-financing trading strategy $\bar{H}$ with associated value process $\bar{V}$ such that $X\left(\omega_{j}\right) \leq \bar{V}\left(T, \omega_{j}\right)$ for every $j=1, \ldots, \kappa$ we have that if $X\left(\omega_{j}\right)<\bar{V}\left(T, \omega_{j}\right)$ for some $j=1, \ldots, \kappa$ then $\bar{V}(0)>V(0)$.

Definition 2.8 We say that a contingent claim $X$ is maximally consistently realizable if $X$ it is consistently realizable and there is a maximal replicating trading strategy $H$ for $X$. 


\section{The single-period case}

In this section we present some results and definitions from the single-period model that we shall use later:

Theorem 3.1 There are no arbitrage opportunities if and only if there exists a probability measure $\pi \in \mathbb{P}(\Omega)$ and a real number $r$ such that

i) $0 \leq r \leq \alpha_{0} E_{\pi}\left[\frac{1}{B(1)}\right]$,

ii) $E_{\pi}\left[\Delta S_{i}^{*}\right] \leq r S_{i}(0) \leq E_{\pi}\left[\Delta S_{i}^{*}+\alpha_{i} \frac{S_{i}(0)}{B(1)}\right]$, for $i=1, \ldots, N$,

iii) $\pi_{j}:=\pi\left(\omega_{j}\right)>0$, for $j=1, \ldots, \kappa$.

Define the matrix $A_{1}$ as:

$$
A_{1}=\left(\begin{array}{cccc}
1 & S_{1}^{*}\left(1, \omega_{1}\right) & \ldots & S_{N}^{*}\left(1, \omega_{1}\right) \\
\vdots & \vdots & \ddots & \vdots \\
1 & S_{1}^{*}\left(1, \omega_{\kappa}\right) & \ldots & S_{N}^{*}\left(1, \omega_{\kappa}\right)
\end{array}\right)
$$

Theorem 3.2 If $B(1)=1+r_{f}$ and $A_{1}$ has an inverse then every contingent claim $X$ is maximally realizable. Moreover there exists a unique maximal trading strategy $H$ that replicates $X$.

Now, define the set $\mathbb{J}:=\left\{a=\left(a_{0}, a_{1}, \ldots, a_{N}\right) ; a_{i}=+\right.$ or -$\}$, and for $a \in \mathbb{J}$, $\operatorname{pos}(a)=\left\{1 \leq i \leq N ; a_{i}=+\right\}$, and $\operatorname{neg}(a)=\left\{1 \leq i \leq N ; a_{i}=-\right\}$. Note that the number of elements of $\mathbb{J}$ is $2^{N+1}$.

Definition 3.3 For $a \in \mathbb{J}$ such that $a_{0}=+$, set

$\Theta^{a}:=\{\pi \in \mathbb{P}(\Omega)$;

a) $\pi_{j}>0, j=1, \ldots, \kappa$,

b) $E_{\pi}\left[\frac{S_{i}(1)}{B(1)}\right]=S_{i}^{*}(0)$ for $i \in \operatorname{pos}(a)$,

c) $E_{\pi}\left[\frac{S_{i}(1)+\alpha_{i} S_{i}(0)}{B(1)}\right]=S_{i}^{*}(0)$ for $\left.i \in \operatorname{neg}(a)\right\}$,

and for $a \in \mathbb{J}$ such that $a_{0}=-$, set

$\Theta^{a}:=\{\pi \in \mathbb{P}(\Omega)$

a) $\pi_{j}>0, j=1, \ldots, \kappa$,

b) $E_{\pi}\left[\frac{S_{i}(1)-\alpha_{0} S_{i}(0)}{B(1)}\right]=S_{i}^{*}(0)$ for $i \in \operatorname{pos}(a)$,

c) $E_{\pi}\left[\frac{S_{i}(1)+\left(\alpha_{i}-\alpha_{0}\right) S_{i}(0)}{B(1)}\right]=S_{i}^{*}(0)$ for $\left.i \in \operatorname{neg}(a)\right\}$.

Theorem 3.4 If $B(1)=1+r_{f}, A_{1}$ has an inverse and for every $a \in \mathbb{J}, \Theta^{a} \neq \emptyset$ then every contingent claim $X$ is maximally consistently realizable. Moreover the maximal replicating trading strategy $H$ is unique.

\section{The multi-period case}

For the multi-period case we follow the approach adopted in [11] by considering the information structure described by the sequence $\mathcal{P}_{0}, \mathcal{P}_{1}, \ldots, \mathcal{P}_{\mathcal{T}}$ of partitions of $\Omega$, with $\mathcal{P}_{0}=\{\Omega\}, \mathcal{P}_{\mathcal{T}}=\left\{\left\{\omega_{1}\right\}, \ldots,\left\{\omega_{\kappa}\right\}\right\}$, and satisfying the property that each $\mathcal{A} \in \mathcal{P}_{t}$ is equal to the union of some elements in $\mathcal{P}_{t+1}$ for every $t<T$ (see [11]). Let us write $\mathcal{P}_{t}=\left\{\mathcal{A}(t, 1), \ldots, \mathcal{A}\left(t, l_{t}\right)\right\}$, and we recall that $\mathcal{A}(t, i) \cap$ $\mathcal{A}(t, j)=\emptyset, i \neq j$ and $\cup_{j=\ell}^{l_{t}} \mathcal{A}(t, \ell)=\Omega$. 
For each $\mathcal{A}(t, \ell)$ let $\nu(t, \ell)$ be the number of sets $\mathcal{A}(t+1, j)$ such that $\mathcal{A}(t+$ $1, j) \subseteq \mathcal{A}(t, \ell)$ (recall that $\mathcal{A}(t, \ell)$ is the union of some elements in $\mathcal{P}_{t+1}$ ). For each $\mathcal{A}(t+1, j)$ such that $\mathcal{A}(t+1, j) \subseteq \mathcal{A}(t, \ell)$, consider a representative element $\omega \in \mathcal{A}(t+1, j)$, and define the set $\Lambda(t, \ell)$ formed by these elements. We set

$$
\Lambda(t, \ell):=\left\{\varpi_{1}(t, \ell) \ldots, \varpi_{\nu(t, \ell)}(t, \ell)\right\} .
$$

The following result can be proved following the same steps as in Pliska [11], pages 95-96, in conjunction with Theorem 3.1 but, we shall omit the details.

Theorem 4.1 There are no arbitrage opportunities if and only if there exists a probability measure $\pi$ and real number $r(t, \ell), t=0, \ldots, T-1, \ell=1, \ldots, l_{t}$, such that for every $\omega \in \mathcal{A}(t, \ell)$,

i) $0 \leq r(t, \ell) \leq \alpha_{0} E_{\pi}\left[\frac{1}{B(t+1)} \mid \mathcal{F}_{t}\right](\omega)$,

ii) for $i=1, \ldots, N$,

$$
\begin{aligned}
& E_{\pi}\left[\Delta S_{i}^{*}(t+1) \mid \mathcal{F}_{t}\right](\omega) \leq r(t, \ell) S_{i}(t)(\omega), \\
& \quad \leq E_{\pi}\left[\Delta S_{i}^{*}(t+1)+\alpha_{i} \frac{S_{i}(t)}{B(t+1)} \mid \mathcal{F}_{t}\right](\omega),
\end{aligned}
$$

iii) $\pi_{j}:=\pi\left(\omega_{j}\right)>0$, for $j=1, \ldots, \kappa$.

For each $t=0, \ldots, T-1$ and $\ell=1, \ldots, l_{t}$, define the matrices

$$
\begin{aligned}
& A_{1}(t, \ell)= \\
& \left(\begin{array}{cccc}
1 & S_{1}^{*}\left(1, \varpi_{1}(t, \ell)\right) & \ldots & S_{N}^{*}\left(1, \varpi_{1}(t, \ell)\right) \\
\vdots & \vdots & \ddots & \vdots \\
1 & S_{1}^{*}\left(1, \varpi_{\nu(t, \ell)}(t, \ell)\right) & \ldots & S_{N}^{*}\left(1, \varpi_{\nu(t, \ell)}(t, \ell)\right)
\end{array}\right) .
\end{aligned}
$$

We have the following result, extending Theorem 3.2 to the multi-period case.

Theorem 4.2 If for every $t=0, \ldots, T-1$ and $\ell=1, \ldots, l_{t}$ we have $B(t+$ $1, \omega)=1+r_{f}(t, \ell)$ for every $\omega \in \mathcal{A}(t, \ell)$, and $A_{1}(t, \ell)$ has an inverse, then every contingent claim $X$ is maximally realizable. Moreover there exists a unique maximal trading strategy $H$ that replicates $X$.

Proof. The basic idea in this proof is to move backward in time from $t=T$ to $t=0$, and apply Theorem 3.2 for each single period $t$ to $t+1$ and each node $\mathcal{A}(t, \ell), \ell=1, \ldots, l_{t}$. From eqn (2) we have

$$
\begin{aligned}
X^{*}=V^{*}(T) & =\left(H_{0}^{+}(T)-H_{0}^{-}(T)\right)+\sum_{i=1}^{N}\left(H_{i}^{+}(T)-H_{i}^{-}(T)\right) S_{i}^{*}(T), \\
& -\left(\alpha_{0} H_{0}^{-}(t) \frac{B(T-1)}{B(T)}+\sum_{i=1}^{N} \alpha_{i} H_{i}^{-}(T) \frac{S_{i}(T-1)}{B(T)}\right) .
\end{aligned}
$$

Applying Theorem 3.2 for each one single period node $\ell=1, \ldots, l_{T-1}$, and recalling that $B(T, \omega)=1+r_{f}(T-1, \ell)$ for every $\omega \in \mathcal{A}(T-1, \ell)$, we obtain 
a unique maximal trading strategy $H(T)$ such that verifies (7). To have a selffinancing trading strategy, we evaluate from (4)

$$
\begin{aligned}
& V(T-1)=\left(H_{0}^{+}(T)-H_{0}^{-}(T)\right) B(T-1), \\
& +\sum_{i=1}^{N}\left(H_{i}^{+}(T)-H_{i}^{-}(T)\right) S_{i}(T-1) .
\end{aligned}
$$

By doing this, we obtain the value of $V(T-1)$ for each node $\ell=1, \ldots, l_{T-1}$. We repeat now the procedure as in (7) to obtain a unique maximal trading strategy $H(T-1)$ that maximally replicates $V(T-1)$, and as in (8) to obtain the values of $V(T-2)$ for each node $\ell=1, \ldots, l_{T-2}$. We carry on doing this up to time $t=0$,

For each $t=0, \ldots, T-1, \ell=1, \ldots, l_{t}$, define $\Theta^{a}(t, \ell)$ as in Definition 3.3, replacing $\mathbb{P}(\Omega)$ by $\mathbb{P}(\mathcal{A}(t, \ell)), S_{i}(1)$ by $S_{i}(t+1)$, and $S_{i}(0)$ by $S_{i}(t)$. We have the following result, extending Theorem 3.4 to the multi-period case.

Theorem 4.3 If for every $t=0, \ldots, T-1$ and $\ell=1, \ldots, l_{t}$ we have $B(t+$ $1, \omega)=1+r_{f}(t, \ell)$ for every $\omega \in \mathcal{A}(t, \ell), A_{1}(t, \ell)$ has an inverse, and for every $a \in \mathbb{J}, \Theta^{a}(t, \ell) \neq \emptyset$ then every contingent claim $X$ is maximally consistently realizable. Moreover there exists a unique maximal self-financing trading strategy $H$ that replicates $X$.

Proof. Following the same idea as in the proof of Theorem 4.2, we move backward in time from $t=T$ to $t=0$, and apply Theorem 3.4 for each one single period node $\ell=1, \ldots, l_{t}$. For the single period $t=T-1$ to $t=T$ and each node $\ell=1, \ldots, l_{T-1}$, we have from Theorem 3.4 that $X$ is maximally consistently realizable. From (8) we get the values of $V(T-1)$ so that the strategy is self-financing. By repeating the same procedure for the single period $t=T-2$ to $t=T-1$ and each node $\ell=1, \ldots, l_{T-2}$, we obtain from Theorem 3.4 that $V(T-1)$ is maximally consistently realizable. We carry on doing this up to the last single period $t=0$ to $t=1$.

Under the assumptions of Theorem 4.3 we have that there will be a seller price and a buyer price for each contingent claim. The seller price, denoted by $V_{s}(0)$, is obtained by applying to $X$ the backward algorithm as presented in Theorem 4.2. The buyer price, denoted by $V_{b}(0)$, is obtained by applying the backward algorithm to $-X$, and taking $V_{b}(0)=-V(0)$.

Let us call $X_{s}(0)$ and $X_{b}(0)$ the seller and buyer prices respectively of $X$ at time $t=0$. If $X_{s}(0)>V_{s}(0)$ then one could sell the contract in the market at the price $X_{s}(0)$, and buy a replicant portfolio at the value $V_{s}(0)$, making a risk-free profit of $X_{s}(0)-V_{s}(0)$. At the final time $T$ the portfolio will provide exactly the right value to settle the obligation on the contingent claim. Thus we have shown that if the market seller price $X_{s}(0)$ is bigger than $V_{s}(0)$, there will exist an arbitrage opportunity. Moreover, from the fact that $X$ is consistently realizable and has a unique replicant trading strategy, no other portfolio will have a final value greater or equal to $X$ with a initial value lower than $V_{s}(0)$. Thus the pricing $V_{s}(0)$ is logically consistent.

A similar conclusion can be constructed to the case $X_{b}(0)<V_{b}(0)$. 
If $V_{b}(0)<X_{b}(0) \leq X_{s}(0)<V_{s}(0)$ then no arbitrage nor perfect hedging can be made.

\section{Example}

Let us consider the binomial model, which consists of a single risky security satisfying $S(t)=u^{N(t)} d^{t-N(t)} S(0), t=1, \ldots, T$, where $0<d<1<u$ and $N=\{N(t) ; t=1, \ldots, T\}$ is a binomial process with parameter $p, 0<$ $p<1$. The interest is assumed to be constant, so that $B(t)=\left(1+r_{f}\right)^{t}, t=$ $0,1, \ldots, T$. Let us obtain conditions that guarantee that every contingent claim $X$ is maximally consistently realizable. It is easy to see that in this case $\mathbb{J}=$ $\{(+,+),(+,-),(-,+),(-,-)\}$, and we have the following possibilities.

i) $(+,+)$; in this case,

$$
\pi_{1}=\frac{1+r_{f}-d}{u-d}, \pi_{2}=\frac{u-\left(1+r_{f}\right)}{u-d} .
$$

ii) $(+,-)$; in this case,

$$
\pi_{1}=\frac{1+r_{f}-\alpha_{1}-d}{u-d}, \pi_{2}=\frac{u-\left(1+r_{f}-\alpha_{1}\right)}{u-d} .
$$

iii) $(-,+)$; in this case,

$$
\pi_{1}=\frac{1+r_{f}+\alpha_{0}-d}{u-d}, \pi_{2}=\frac{u-\left(1+r_{f}+\alpha_{0}\right)}{u-d} .
$$

iv) $(-,-)$; in this case,

$$
\pi_{1}=\frac{1+r_{f}+\alpha_{0}-\alpha_{1}-d}{u-d}, \pi_{2}=\frac{u-\left(1+r_{f}+\alpha_{0}-\alpha_{1}\right)}{u-d} .
$$

From above it is clear that the condition which guarantees that every contingent claim $X$ is maximally consistently realizable is that $u>1+r_{f}+\alpha_{0}$ and $d<$ $1+r_{f}-\alpha_{1}$. If this is satisfied, we have $0<\pi_{1}<1,0<\pi_{2}<1$ for all the four cases above.

Let us consider the following numerical example. Suppose that $S(0)=5, u=$ $\frac{4}{3}, d=\frac{8}{9}, \alpha_{0}=\alpha_{1}=\frac{1}{30}, r_{f}=\frac{1}{9}$. For this case we have $1+r_{f}+\alpha_{0}=$ $\frac{103}{90}<u=\frac{4}{3}$, and $1+r_{f}-\alpha_{1}=\frac{97}{90}>d=\frac{8}{9}$, and every contingent claim $X$ is maximally consistently realizable. Let us consider the following option: $X=$ $\max \{S(2)-5,0\}$. By applying the backward procedure described in Theorem 4.2 we obtain that the seller price for $X$ is $V_{s}(0)=1.3272$, with the following heading strategy: $H_{0}^{+}(0)=0, H_{0}^{-}(0)=2.796, H_{1}^{+}(0)=0.8246, H_{1}^{-}(0)=0$, and for the case in which the risky security goes up, $H_{0}^{+}(1)=0, H_{0}^{-}(1)=3.932$, $H_{1}^{+}(1)=1.0, H_{1}^{-}(1)=0, V(1)=2.2977$, while for the case in which it goes down, $H_{0}^{+}(0)=0, H_{0}^{-}(0)=1.4563, H_{1}^{+}(0)=0.4687, H_{1}^{-}(0)=0$ and $V(1)=$ 0.4652 . 
By repeating the procedure now for $-X$ obtain that the buyer price for $X$ is $V_{b}(0)=0.9355$, with the following heading strategy: $H_{0}^{+}(0)=2.6926, H_{0}^{-}(0)=$ $0, H_{1}^{+}(0)=0, H_{1}^{-}(0)=0.7256$, and for the case in which the risky security goes up, $H_{0}^{+}(1)=4.23, H_{0}^{-}(1)=0, H_{1}^{+}(1)=0, H_{1}^{-}(1)=1.0, V(1)=1.9667$, while for the case in which it goes down, $H_{0}^{+}(0)=1.5562, H_{0}^{-}(0)=0, H_{1}^{+}(0)=$ $0, H_{1}^{-}(0)=0.4688$ and $V(1)=0.3542$. As expected, $V_{b}(0)=0.9355<V_{s}(0)=$ 1.3272 .

\section{Acknowledgment}

This work was partially supported by CNPq (Brazilian National Research Council), grants 472920/03-0 and 304866/03-2, FAPESP (Research Council of the State of São Paulo), grant 03/06736-7, PRONEX, grant 015/98, and IM-AGIMB.

\section{References}

[1] Edirisinghe, C., Naik, V. \& Uppal, R., Optimal replication of options with transactions costs and trading restrictions. Journal of Financial and Quantitative Analysis, 28(1), pp. 117138, 1993.

[2] Davis, M.H.A., Panas, V.G. \& Zariphopoulou, T., European option pricing with transactions costs. SIAM J Control Optim, 34, pp. 470493, 1993.

[3] Leland, H.E., Option pricing and replication with transactions costs. The Journal of Finance, 40(5), pp. 12831301, 1985.

[4] Boyle, P.P. \& Vorst, T., Option replication in discrete time with transactions costs. The Journal of Finance, 47(1), pp. 271293, 1992.

[5] Cox, J.C., Ross, S.A. \& Rubinstein, M., Option pricing: A simplified approach. Journal of Financial Economics, 7, pp. 229263, 1979.

[6] Melnikov, A.V. \& Petrachenko, Y.G., On option pricing in binomial market with transaction costs. Finance and Stochastics, 9, pp. 141149, 2005.

[7] Bertsimas, D., Kogan, L. \& Lo, A.W., Hedging derivative securities and incomplete markets: An $\epsilon$ arbitrage approach. Operations Research, 49(3), pp. 372397, 2001.

[8] Liu, H., Optimal consumption and investment with transactions costs and multiple risky assets. The Journal of Finance, 49(1), pp. 289331, 2004.

[9] Cvitanic, J., Minimizing expected loss of hedging in incomplete and constrained markets. SIAM J Control Optim, 38(4), pp. 10501066, 2000.

[10] Stettner, L., Option pricing in discrete-time incomplete market models. Mathematical Finance, 10, pp. 305321, 2000.

[11] Pliska, S.R., Introduction to Mathematical Finance. Blackwell Publishers, 1997. 